\title{
ARTICLE OPEN \\ Risk of second primary malignancies in head and neck cancer patients treated with definitive radiotherapy
}

Sweet Ping Ng ${ }^{1,2 *}$, Courtney Pollard $\mathrm{III}^{1}$, Mona Kamal ${ }^{1}$, Zeina Ayoub ${ }^{1}$, Adam S. Garden ${ }^{1}$, Houda Bahig ${ }^{1}$, G. Brandon Gunn ${ }^{1}$, Steven J. Frank ${ }^{1}$, Heath D. Skinner $\mathbb{D}^{1}$, Jack Phan ${ }^{1}$, Joel Berends ${ }^{3}$, William H. Morrison ${ }^{1}$, Jason M. Johnson ${ }^{4}$, Renata Ferrarotto ${ }^{5}$, Erich M. Sturgis ${ }^{6}$, Abdallah S. R. Mohamed (D) ${ }^{1}$, Stephen Y. Lai ${ }^{6}$, Clifton D. Fuller (D) ${ }^{1}$ and David I. Rosenthal ${ }^{1 *}$

Second primary malignancy (SPM) may occur after index head and neck cancer (HNC) treatment. This study evaluated the prevalence and outcome of SPM in patients with HNC treated with definitive radiotherapy. Eligible patients include those with index mucosal HNC treated with definitive radiotherapy between 2000 and 2010. SPM was defined as an invasive cancer at a noncontiguous site diagnosed at least 6 months after completion of radiotherapy. Clinical data were collected, and the Kaplan-Meier method was used to estimate overall survival. In total, 1512 patients were studied. The majority of patients had index oropharyngeal cancer (86\%). In all, 130 (9\%) patients developed a SPM. The risk of SPM increased exponentially with time with 5-, $10-$, and 15 -year rates of 4,10 , and $25 \%$. Half of SPMs were within the head and neck or thoracic regions. SPM rates were significantly higher $(p<0.0001)$ in current smokers and former smokers than never smokers with 5-, 10-, and 15-year risk being: never smoker $(2,4,14 \%)$, former smokers with <10-pack year $(5,10,23 \%)$, former smokers with $\geq 10$-pack year $(5,14,35 \%)$, and current smokers $(6,18,32 \%)$. In total, $102(78 \%)$ had subsequent curative-intent therapy. The 5-year overall survival from SPM was $44 \%$. The majority of SPMs were in those with significant smoking history reflecting the same risk factor as for the index mucosal HNC. Nearly one in two patients with SPMs were salvaged underscoring the importance of regular surveillance for SPMs.

npj Precision Oncology (2019)3:22

; https://doi.org/10.1038/s41698-019-0097-y

\section{INTRODUCTION}

Head and neck squamous cell carcinoma (HNSCC) has an incidence of $\sim 50,000$ annual cases in the United States, with an annual mortality estimated at 10,000 persons. ${ }^{1}$. With advancements in diagnosis and treatment, the number of head and neck cancer survivors is increasing. The 5-year overall survival for patients with head and neck mucosal cancer treated with radiotherapy is estimated at $65 \%$ according to the SEER data from 2008 to 2014 . $^{2}$ In the post treatment surveillance setting, we monitor for disease recurrence, late effects of treatment, and second primary cancer. As the risk of index HNSCC disease recurrence decreases exponentially over time, survivorship care focuses on prevention and management of late effects of therapy, and prevention and early detection of second primary malignancy (SPM). SPM may occur after index primary head and neck cancer treatment. Previous studies have reported the incidence of SPMs in a mixed cohort of patients with head and neck cancer treated with or without radiotherapy. The risk of SPM in patients with index head and neck cancer treated with definitive radiotherapy in the contemporary era has not been well documented. Here, we report the prevalence of SPM and subsequent outcomes of patients with SPMs in a large cohort of patients with mucosal squamous cell carcinoma of the head and neck treated with curative-intent radiotherapy.

\section{RESULTS}

Patient characteristics

From year 2000 to 2010,1512 patients were eligible for analysis. The median age of the cohort was 55 years (range: $14-87$ years).
The most common index tumor site was the oropharynx (86\%), followed by the larynx (7\%). The median radiation dose and fractions delivered were $6996 \mathrm{cGy}$ and 33 fractions. The majority of patients $(89 \%)$ were treated using intensity-modulated radiation therapy technique. Patients' and treatment characteristics of the cohort are summarized in Table 1.

Risk of development of a second primary

One hundred and thirty (9\%) patients developed a SPM, with 114 patients diagnosed with SPM at least 2 years post treatment. The median time to develop a SPM was 72 months (range: 6-178 months). As depicted in Fig. 1, the actuarial risk of SPM increased exponentially with time with overall rates at $2,5,10$, and 15 years were $1,4,10.3$, and $25.2 \%$, respectively.

There was no significant difference in the proportion of patients who developed a SPM according to radiation treatment technique, with $18(14 \%)$ patients previously treated with 3D conformal technique and 112 (86\%) had IMRT.

\section{Smoking status and risk of SPM}

Of the 130 patients who had a SPM, 90 (70\%) had significant smoking history with 42 current smoker and 48 with $\geq 10$-pack year smoking history at the diagnosis of index head and neck carcinoma. Overall, 23 patients were never smoker and 65 were former smoker (17 had <10-pack year history, and 48 had $\geq 10$ pack year smoking history). SPM rates were significantly higher ( $p$ $<0.0001)$ in patients who were current smokers and former

\footnotetext{
1Department of Radiation Oncology, Division of Radiation Oncology, The University of Texas MD Anderson Cancer Center, Houston, TX, USA. ${ }^{2}$ Department of Radiation Oncology, Peter MacCallum Cancer Centre, Melbourne, Victoria, Australia. ${ }^{3}$ The University of Texas Health Science Center, San Antonio, TX, USA. ${ }^{4}$ Department of Diagnostic Radiology, The University of Texas MD Anderson Cancer Center, Houston, TX, USA. ${ }^{5}$ Department of Thoracic Head and Neck Medical Oncology, Division of Cancer Medicine, The University of Texas MD Anderson Cancer Center, Houston, TX, USA. ${ }^{6}$ Department of Head and Neck Surgery, Division of Surgery, The University of Texas MD Anderson Cancer Center, Houston, TX, USA. *email: sweet.ng@petermac.org; dirosenthal@mdanderson.org
} 
Table 1. Patients and treatment characteristics

\begin{tabular}{|c|c|c|c|}
\hline Parameters & $N(n=1512)$ & $\%$ & SPM (\%) \\
\hline Age & \multicolumn{3}{|c|}{$\begin{array}{l}\text { Median }=55 \text { years (range: } \\
14-87 \text { years) }\end{array}$} \\
\hline \multicolumn{4}{|l|}{ Gender } \\
\hline Male & 1285 & 84.9 & 105 (6.9) \\
\hline Female & 227 & 15 & $25(1.7)$ \\
\hline \multicolumn{4}{|l|}{ Primary site } \\
\hline Oropharynx & 1300 & 86 & $108(7.2)$ \\
\hline Larynx & 105 & 6.9 & $13(0.9)$ \\
\hline Nasopharynx & 87 & 5.8 & $6(0.4)$ \\
\hline Sinonasal & 12 & 0.8 & $1(0.1)$ \\
\hline Oral cavity & 8 & 0.5 & $2(0.1)$ \\
\hline \multicolumn{4}{|l|}{$T$ category } \\
\hline $\mathrm{T} 1$ & 396 & 26.2 & $28(1.9)$ \\
\hline $\mathrm{T} 2$ & 520 & 34.4 & $53(3.5)$ \\
\hline T3 & 310 & 20.5 & $30(2.0)$ \\
\hline T4 & 231 & 15.3 & $16(1.1)$ \\
\hline Tx & 55 & 3.6 & $3(0.2)$ \\
\hline \multicolumn{4}{|l|}{$N$ category (all except nasopharynx) } \\
\hline No & 172 & 11.4 & $17(1.1)$ \\
\hline N1 & 183 & 12.1 & $19(1.3)$ \\
\hline $\mathrm{N} 2 \mathrm{a}$ & 132 & 8.7 & $8(0.5)$ \\
\hline $\mathrm{N} 2 \mathrm{~b}$ & 574 & 38 & $51(3.4)$ \\
\hline $\mathrm{N} 2 \mathrm{c}$ & 219 & 14.5 & $17(1.1)$ \\
\hline N3 & 101 & 6.7 & $8(0.5)$ \\
\hline $\mathrm{Nx}$ & 44 & 2.9 & $4(0.3)$ \\
\hline \multicolumn{4}{|l|}{ N category (nasopharynx) } \\
\hline No & 16 & 1.1 & 0 \\
\hline N1 & 22 & 1.5 & $19(1.3)$ \\
\hline N2 & 37 & 2.5 & $3(0.2)$ \\
\hline N3 & 12 & 0.8 & 0 \\
\hline \multicolumn{4}{|l|}{ AJCC stage } \\
\hline I & 61 & 4 & $7(0.5)$ \\
\hline II & 104 & 6.9 & $10(0.7)$ \\
\hline III & 234 & 15.5 & $25(1.7)$ \\
\hline IV & 1113 & 73.6 & $88(5.8)$ \\
\hline \multicolumn{4}{|l|}{ Smoking status at diagnosis } \\
\hline Current & 368 & 24.3 & $42(2.8)$ \\
\hline Ex-smoker ( $\geq 10$-pack years) & 408 & 27 & $48(3.2)$ \\
\hline Ex-smoker (<10-pack years) & 199 & 13.2 & $17(1.1)$ \\
\hline Never smoker & 537 & 35.5 & $23(1.5)$ \\
\hline \multicolumn{4}{|l|}{ Radiotherapy technique } \\
\hline 3D conformal & 172 & 11.4 & $18(1.2)$ \\
\hline IMRT & 1340 & 88.6 & $112(7.4)$ \\
\hline Radiation dose & \multicolumn{3}{|c|}{$\begin{array}{l}\text { Median }=6996 \text { cGy (range: } \\
5710-7600 \text { cGy) }\end{array}$} \\
\hline Number of radiotherapy fractions & \multicolumn{3}{|c|}{ Median $=33$ (range: $28-64)$} \\
\hline \multicolumn{4}{|l|}{ Induction chemotherapy } \\
\hline Yes & 446 & 29.5 & $32(2.1)$ \\
\hline No & 1066 & 70.5 & $98(6.5)$ \\
\hline \multicolumn{4}{|l|}{ Concurrent chemotherapy } \\
\hline Yes & 1005 & 66.5 & $89(5.9)$ \\
\hline No & 507 & 33.5 & $41(2.7)$ \\
\hline
\end{tabular}

$3 D$ three-dimensional, IMRT intensity-modulated radiation therapy $\mathrm{T}$ and $\mathrm{N}$ categories were classified according to the AJCC 7th edition

smokers than never smokers (Fig. 2). The cumulative rates of SPM for each smoking group at 2, 5, 10, and 15 years were: never smokers $(1,2,4,14 \%)$, former smokers with < 10-pack year $(2,5,10$, $23 \%)$, former smokers with $\geq 10$-pack year $(1,5,14,35 \%)$, and current smokers $(1,6,18,32 \%)$, respectively (Fig. 2). Similar pattern

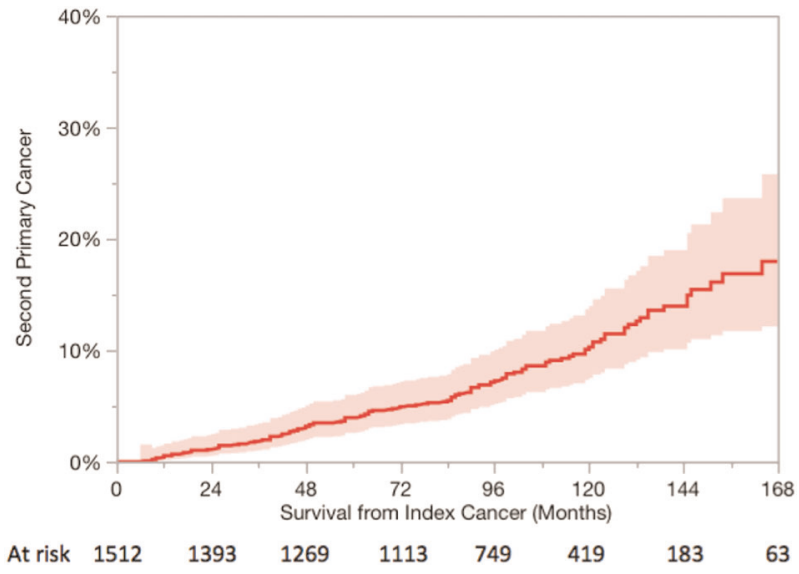

Fig. 1 Actuarial risk of developing a second primary malignancy after completion of definitive radiotherapy for index mucosal head and neck squamous cell carcinoma

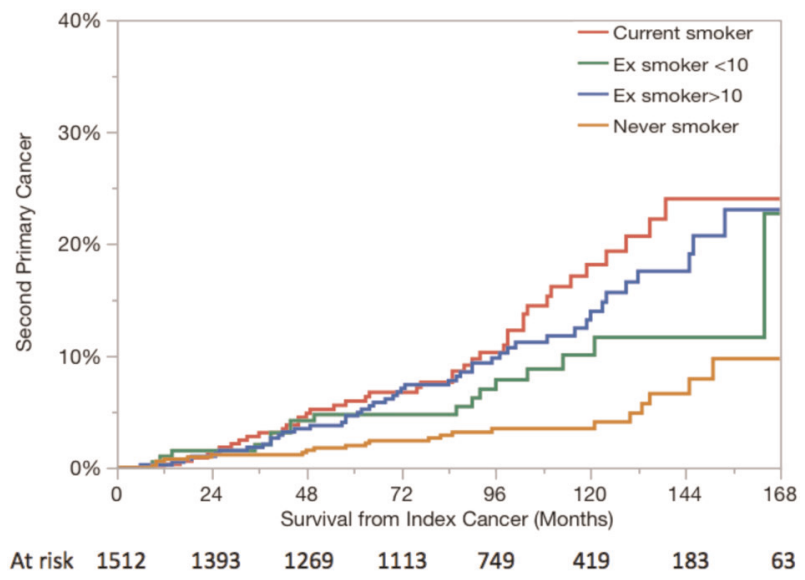

Fig. 2 The actuarial risk of SPM according to smoking status at diagnosis of index head and neck cancer

was observed in those with index oropharyngeal cancer with 2-, $5-, 10-$, and 15 -year cumulative rates of SPM of $0,1,5$, and $25 \%$ for never smokers and former smokers with $<10$-pack year, and 2, 11, 10 , and $42 \%$ ) for those who were current smokers and former smokers with > 10-pack year (Fig. 3). On multivariate analysis, patient's age and smoking status at diagnosis of index cancer and the use of induction chemotherapy emerged as risk factors for the development of SPM (Table 2).

Sites of SPM

Figure 4 displays the sites and frequencies of SPMs in this cohort. Almost half of the cohort (49\%) subsequently developed a SPM within the head and neck, or the upper aerodigestive tract regions -36 (28\%) had a second mucosal head and neck carcinoma, 2 (2\%) had sarcoma of the head and neck, and 26 (20\%) had esophageal (5 patients) or lung carcinoma (21 patients). In those with SPM, 107 (82\%) patients were current or former smokers -42 current, 48 former with $\geq 10$-pack year, and 17 former with $<10$ pack year. Patients who had significant smoking consumption (current and those with $\geq 10$-pack year) comprised $92 \%$ of thoracic and $61 \%$ of head and neck SPMs.

Genitourinary cancer was the second most common cancer in this group - 23 prostate cancers, 3 bladder cancers, 2 renal cancers, 1 urethral cancer, and 1 testicular cancer. Of the five 

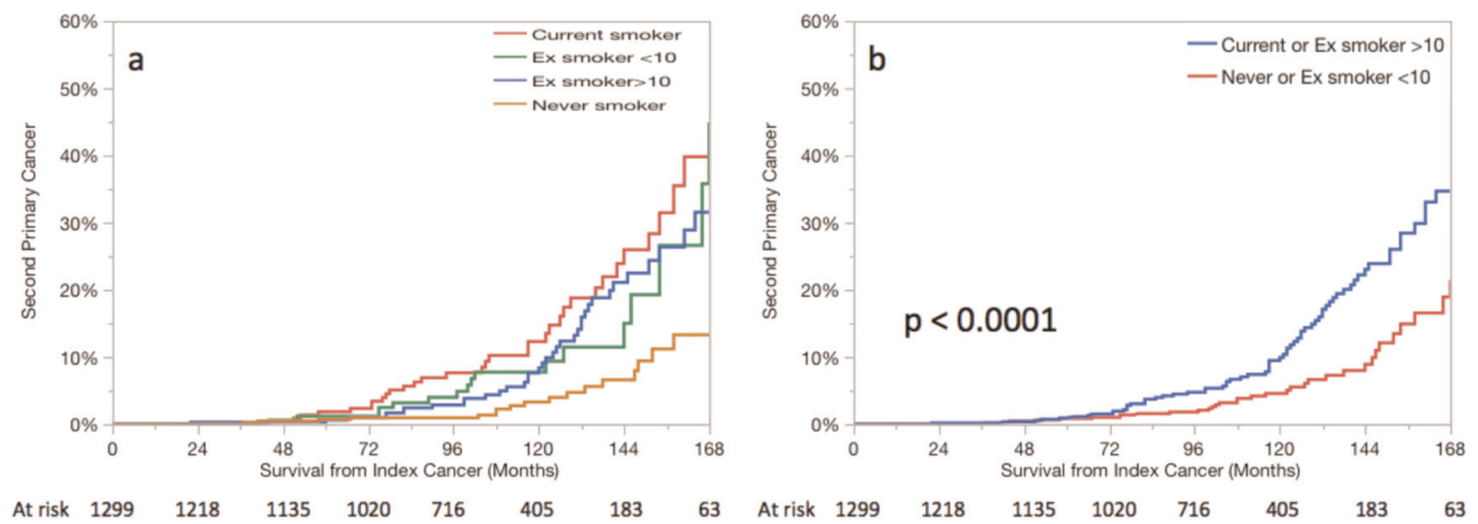

Fig. 3 The actuarial risk of SPM according to smoking status at diagnosis for patients with index oropharyngeal cancer. a Stratified according to smoking status, b stratified into two groups (current or former smoker with $>10$-pack years versus never or former smoker with $<10$ pack years)

Table 2. Multivariate analysis for the risk of development of SPM over time after index cancer

\begin{tabular}{|c|c|c|c|}
\hline Parameters & Risk ratio & $\begin{array}{l}95 \% \text { confidence } \\
\text { interval }\end{array}$ & $p$-value \\
\hline Age & 17.79 & $4.6-68.4$ & $<0.0001$ \\
\hline Sex (male) & 0.7 & $0.47-1.14$ & 0.16 \\
\hline $\begin{array}{l}\text { Smoking status (current or ex } \\
>10 \text { ) }\end{array}$ & 2.14 & $1.47-3.16$ & $<0.0001$ \\
\hline AJCC stage & 1.1 & $0.42-3.14$ & 0.81 \\
\hline $\begin{array}{l}\text { Induction } \\
\text { chemotherapy (yes) }\end{array}$ & 1.96 & $1.25-3.02$ & 0.004 \\
\hline $\begin{array}{l}\text { Concurrent } \\
\text { chemotherapy (yes) }\end{array}$ & 1.46 & $0.97-2.24$ & 0.08 \\
\hline
\end{tabular}

$P$-values $<0.05$ are considered significant

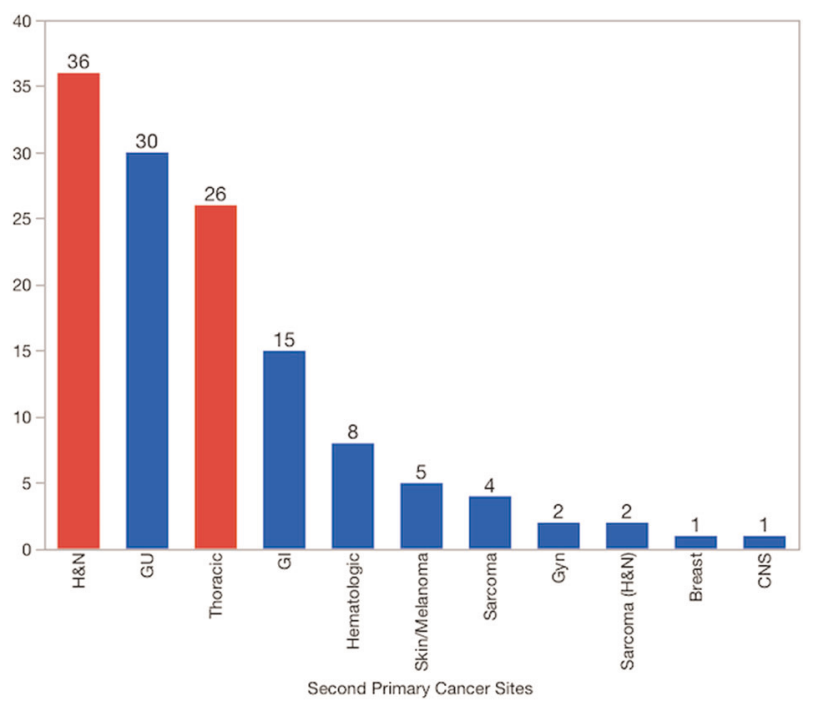

Fig. 4 Sites and frequencies of second primary malignancies. Bars in red indicated SPMs within the head and neck, and upper aerodigestive tract (lung/esophagus)

patients with bladder and renal cancers, four patients were current or former with $\geq 10$-pack year smokers. With regards to HPVrelated cancers outside the head and neck region, one patient developed cervical cancer 26 months after completing radiotherapy for tonsil cancer. Out of the 130 cases of SPMs, 66 were symptomatic and 64 were detected via routine screening examinations and/or investigations. Table 3 showed the distribution of head and neck SPM in relation to index cancer site.

Clinical outcomes of those with SPMs

Overall, the median overall survival for the cohort was 98 (range: 6-199) months. One hundred and two (78\%) patients had subsequent curative-intent therapy for SPM. The 2-year and 5year overall survival from diagnosis of SPM was 67 and 44\% (Fig. 5). There was no significant difference in overall survival according to smoking status. The 2- and 5-year overall survival according to smoking status were 85 and $40 \%$ for never smokers, 82 and $62 \%$ for former smokers with <10-pack year, 64 and $47 \%$ for former smokers with $\geq 10$-pack year, and 57 and $35 \%$ for current smokers.

\section{DISCUSSION}

In this study, we reported the prevalence of SPMs and the outcomes of those who had SPMs in a large cohort of patients with an index HNSCC treated with curative-intent radiotherapy. Our study highlighted that those with active or $\geq 10$-pack year smoking at the diagnosis of index HNSCC were at significant risk of a subsequent SPM compared with former smokers with $<10$ pack year or never smokers. The majority of those with current smoking or former smoking status with $\geq 0$-pack year history with SPMs had a second cancer within the upper aerodigestive tract and thorax. Furthermore, the 5-year overall survival in those who developed SPM was $44 \%$, indicating that effective treatment options are available for this group of patients.

This study showed that there was no difference in the proportion of patients who developed SPMs in those who received radiotherapy via 3D-CRT or IMRT technique. Our finding is consistent with previous studies where the technique of radiation delivery did not correlate with the incidence of second primary malignancies. ${ }^{3}$ However, the radiation technique may be associated with the type of second malignancy where 3D-CRT tend to be associated with the development of in-field sarcoma due to the high-dose radiation received by normal tissues. The induction of sarcoma generally require doses of $48 \mathrm{~Gy}$ or higher with a prolonged latency period of more than 10 years. ${ }^{4}$ IMRT which delivers dose to the target via multiple treatment fields, on the contrary, is believed to be associated with non-sarcoma second malignancies, as there is a higher volume of normal tissues receiving low-radiation dose compared with $3 \mathrm{D}-\mathrm{CRT}{ }^{3,5,6} \mathrm{~A}$ study investigating second cancers secondary to radiotherapy in the US Surveillance, Epidemiology and End Results (SEER) cancer registries showed no significant relative risk of second cancer for 
Table 3. Sites of head and neck SPM compared to index head and neck carcinoma site

\begin{tabular}{lll}
\hline Index cancer site & Primary site of SPM & $\begin{array}{l}\text { Number of cases } \\
(n=36)\end{array}$ \\
\hline Tonsil $(n=18)$ & Oral tongue & 7 \\
& Larynx & 3 \\
& Thyroid & 2 \\
& Base of tongue & 2 \\
& Parotid & 1 \\
& Retromolar trigone & 1 \\
& Tonsil (contralateral) & 1 \\
Base of tongue $(n=14)$ & Larynx & 7 \\
& Oral tongue & 3 \\
& Gingiva & 2 \\
& Base of tongue & $1^{\text {a }}$ \\
Soft palate & Tonsil & 1 \\
Nasopharynx & Retromolar trigone & 1 \\
Maxillary sinus & Oral tongue & 1 \\
Retromolar trigone & Hard palate & $1^{\text {b }}$ \\
\hline
\end{tabular}

${ }^{a}$ Second base of tongue cancer was located away from index tumor location and diagnosed at $>10$ years from the index cancer

${ }^{\mathrm{b}}$ The second tumor was of a different histology than the index cancer

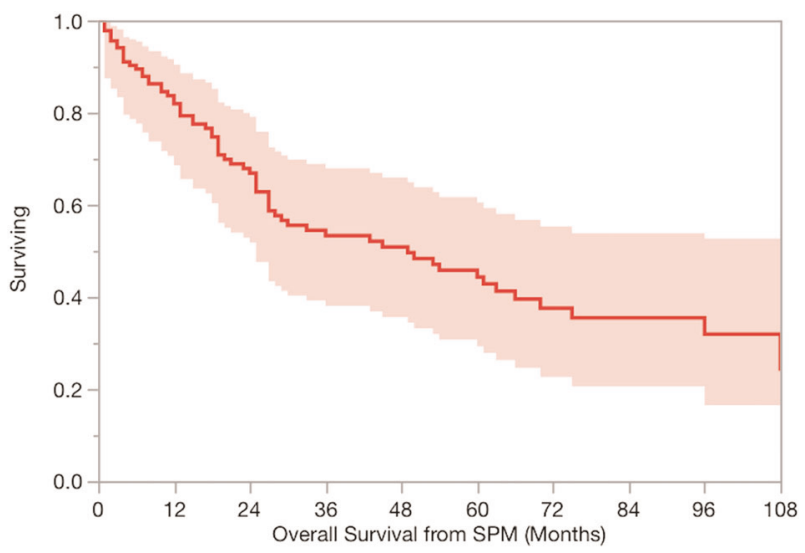

Fig. 5 Overall survival from diagnosis of SPM

patients who received high-dose radiotherapy for an index head and neck cancer. ${ }^{7}$ In another SEER study, Gao et al. ${ }^{8}$ reported that radiotherapy was significantly associated with $10 \%$ increased risk of SPMs in patients with laryngeal cancer, particularly lung cancer. However, this study did not account for smoking status of patients and the location of lung tumors were not described, as radiotherapy fields for laryngeal cancer typically only involved the apices of lungs.

The risk of developing SPMs secondary to radiotherapy remained contentious, as the majority of patients with an index cancer tend to be at higher risk of a second cancer compared with the general population due to their pre-existing risk factors such as lifestyle (smoking, alcohol), environmental, and genetic susceptibilities. As expected, our data clearly reflect the effect of field cancerisation, with almost half of the SPMs occurring within the bronchogenic, esophageal, or head and neck mucosa. In addition, we demonstrated that patients with significant smoking history had higher rates of a second mucosal head and neck, esophageal, or lung cancer. This is of no surprise as the relationship between tobacco usage in patients with index head and neck cancer and subsequent multiple primary cancers has been reported vastly in the literature. ${ }^{9-15}$ Cooper et al. ${ }^{16}$ reported similar findings in 1989 in the Radiation Therapy Oncology Group (RTOG) database, where 110 SPMs were detected in a cohort of 928 patients, with $64 \%$ of SPMs arising from the upper aerodigestive tract. Although the rate of SPMs reported in our cohort is similar to historical studies, our cohort had improved 5year OS of $44 \%$ compared with historical studies where 5-year OS was $<30 \%{ }^{12,16-18}$ potentially reflecting increased physicians' awareness to screen for SPMs, improved treatment options and efficacy, and success of lifestyle modifications programs, such as tobacco cessation and cancer-screening programs. In addition, cancer chemoprevention is an emerging field, although consensus on the absolute benefit of the drug versus potential side effects of the drug (for example the use of tamoxifen and raloxifene to reduce the absolute risk of breast cancer) remained contentious. ${ }^{19}$ Certain chemoprevention strategies such as the role of aspirin in reducing colorectal cancer risk remained to be proven. However, these options can be discussed and explored with patients at high risk of certain SPM at cancer-screening programs.

More recently, the identification of the human papillomavirus (HPV) as an etiology for oropharyngeal cancer has revolutionized risk stratification for oropharyngeal cancer, with HPV-related oropharyngeal cancer associated with improved survival compared with their non-HPV-associated counterpart. ${ }^{20}$ Although smoking remained a significant risk factor for developing mucosal head and neck cancer, the incidence of the "classic" patient with smoking-related oropharyngeal cancer is on the decline, while the incidence of HPV-related oropharyngeal cancer is on the rise. ${ }^{21-24}$ As those who have HPV-related oropharyngeal cancer tend to be of a younger age demographic, coupled with improved survival, there will be an increase of long-term survivors in several years time. Although HPV/p16 data were not available for our cohort, $\sim 86 \%$ had oropharyngeal cancer and $48 \%$ of the patients were non-smokers or previous light smokers ( $<10$-pack year history), therefore we may be able to deduce that a significant number of these patients were likely to have an index HPV-related oropharyngeal cancer. Morris et $\mathrm{al}^{25}$ demonstrated that among patients with oropharyngeal cancer, the SPM risk has decreased significantly since 1991 assuming that the majority of these cases were HPV related. As the study is based on SEER database, tobacco use and HPV/p16 status were not available.

In addition to the importance of lifestyle modifications and screening for SPMs within the upper respiratory tract, our study highlights the importance of regular cancer screening in patients with head and neck cancer. Although the primary focus of followup after an index head and neck cancer is to monitor the upper aerodigestive tract for recurrence, late effects and SPM, here we showed that a half of our patients develop malignancies beyond the upper aerodigestive tract. Common malignancies such as skin, colorectal, breast, and prostate cancers have effective screening programs. Therefore, we recommend that patients should be referred and enrolled in gender and age-appropriate cancer screening programs. In addition, patients who had a significant tobacco use history should be referred for consideration of lung cancer screening. With early detection and the availability of effective therapies, the cure rates of these SPMs are potentially high. $^{26}$

Our study comes with several caveats. First, there are limitations of a retrospective single institutional study. Second, we reported a strong correlation between smoking status at diagnosis of index HNSCC and subsequent SPM, but we do not have data on the effect of smoking cessation after initial diagnosis on the development of SPM. Third, our study included a heterogenous group of patients and approximately one-third of the group did receive induction chemotherapy. Although our multivariate analysis indicated that induction chemotherapy is a risk factor in 
the development of SPM, we did not have detailed chemotherapy (induction and concurrent) data and therefore, it was difficult to distinguish the effect of specific chemotherapy agents on subsequent development of SPM. In addition, as the cohort was pre-2010, we do not have p16 or HPV data on those with index oropharyngeal cancer. It is possible that the majority of the oropharyngeal cohort had p16/ HPV-related cancer, as approximately half of the cohort was non-smokers or previous light smokers with <10-pack year smoking history. As the routine reporting of p16 and/or HPV status occurs after the year 2010, and the median time for the emergence of a SPM is $\sim 6$ years after completion of treatment for index HNSCC, we are unlikely to have "real-life" data for many more years. Finally, another caveat of the study is that we report on prevalence of SPMs after completion of radiotherapy and some SPM that emerge within the first 2 years after treatment could have been present during index HNSCC diagnosis and treatment at a microscopic level. Nonetheless, to our knowledge, our study is the largest single institutional cohort study that provided the data on the prevalence of SPMs and subsequent outcomes in those who had definitive radiotherapy for index HNSCC.

In this primarily IMRT population, the majority of SPMs were in those with an active smoking status or former smokers with $\geq 10$ pack year history. This study highlights the importance of regular clinical surveillance for SPMs in this population, as almost half of those with SPM can be successfully treated. In addition, tobacco cessation advocacy efforts should continue, and age and/or gender-appropriate cancer screening should not be overlooked in this group of patients during follow-up. In the "HPV epidemic" era, focus on identifying SPM and cancer screening during follow-up is particularly important for those with HPV-related oropharyngeal cancer, as this subgroup of patients have good prognosis and long-term survival.

\section{METHODS}

Eligible cases included those treated with definitive radiotherapy for an index head and neck mucosal squamous cell carcinoma at The University of Texas MD Anderson Cancer Center, USA between year 2000 and 2010. SPM was defined as an invasive solid cancer at a noncontiguous site or a hematologic malignancy diagnosed at least 6 months after completion of initial definitive radiotherapy course. This is similar to the Warren and Gates ${ }^{27}$ criteria for multiple primary malignancies:

1. The tumor must be clearly malignant on histology.

2. The tumor must be geographically and/or histopathologically distinct from the index tumor.

3. The possibility of second tumor being a metastasis from the index tumor must be excluded.

Clinical, treatment, and disease data including age at diagnosis, index tumor site and stage, use of induction chemotherapy, radiation dose and fractionation, site of second primary, and subsequent treatment intent of SPM were recorded. Smoking status and tobacco history at presentation of initial cancer diagnosis were also collected. Human papillomavirus (HPV) and p16 status were not routinely reported prior to 2010, and therefore were not collected. This retrospective study was approved by the Institutional Review Board (IRB).

\section{Statistical analysis}

The actuarial risk of developing a SPM was calculated from date of completion of radiotherapy for the index cancer to the date of second primary diagnosis. Overall survival from SPM was determined from the date of second primary diagnosis to date of death from any cause, or date last known alive. Survival outcomes were estimated using the Kaplan-Meier method and groups (smoking status) were compared using the log-rank test. Univariate and multivariate analyses were performed. $P$-value of 0.05 or less was considered statistically significant. All statistical analyses were performed using the JMP package (v 12.1.0, SAS Institute Inc.).
Reporting summary

Further information on research design is available in the Nature Research Reporting Summary linked to this article.

\section{DATA AVAILABILITY}

The data set generated during and/or analyzed during the current study is available from the corresponding author on reasonable request.

Received: 23 June 2019; Accepted: 10 September 2019; Published online: 27 September 2019

\section{REFERENCES}

1. Siegel, R. L., Miller, K. D. \& Jemal, A. Cancer statistics, 2017. CA Cancer J. Clin. 67 7-30 (2017).

2. SEER Cancer Statistics Review (eds Noone, A. M. et al.). https://seer.cancer.gov/ csr/1975 2015/. (National Cancer Institute, Bethesda, MD, 1975-2015).

3. Hall, E. J. \& Wuu, C. S. Radiation-induced second cancers: the impact of 3D-CRT and IMRT. Int. J. Radiat. Oncol. Biol. Phys. 56, 83-88 (2003).

4. Tubiana, M. Can we reduce the incidence of second primary malignancies occurring after radiotherapy? A critical review. Radiother. Oncol. 91, 4-15 (2009).

5. Hall, E. J. Intensity-modulated radiation therapy, protons, and the risk of second cancers. Int. J. Radiat. Oncol. Biol. Phys. 65, 1-7 (2006).

6. Kry, S. F. et al. The calculated risk of fatal secondary malignancies from intensitymodulated radiation therapy. Int. J. Radiat. Oncol. Biol. Phys. 62, 1195-1203 (2005).

7. Berrington de Gonzalez, A. et al. Proportion of second cancers attributable to radiotherapy treatment in adults: a cohort study in the US SEER cancer registries. Lancet Oncol. 12, 353-360 (2011).

8. Gao, X., Fisher, S. G., Mohideen, N. \& Emami, B. Second primary cancers in patients with laryngeal cancer: a population-based study. Int. J. Radiat. Oncol. Biol. Phys. 56, 427-435 (2003).

9. Wynder, E. L., Mushinski, M. H. \& Spivak, J. C. Tobacco and alcohol consumption in relation to the development of multiple primary cancers. Cancer 40, 1872-1878 (1977).

10. Goodner, J. T. \& Watson, W. L. Cancer of the esophagus; its association with other primary cancers. Cancer 9, 1248-1252 (1956).

11. Sturgis, E. M. \& Miller, R. H. Second primary malignancies in the head and neck cancer patient. Ann. Otol. Rhinol. Laryngol. 104, 946-954 (1995).

12. Schwartz, L. H. et al. Synchronous and metachronous head and neck carcinomas. Cancer 74, 1933-1938 (1994).

13. Dikshit, R. P. et al. Risk factors for the development of second primary tumors among men after laryngeal and hypopharyngeal carcinoma. Cancer 103, 2326-2333 (2005).

14. Day, G. L. \& Blot, W. J. Second primary tumors in patients with oral cancer. Cancer 70, 14-19 (1992).

15. Leon, X. et al. Second neoplasm in patients with head and neck cancer. Head Neck 21, 204-210 (1999).

16. Cooper, J. S. et al. Second malignancies in patients who have head and neck cancer: incidence, effect on survival and implications based on the RTOG experience. Int. J. Radiat. Oncol. Biol. Phys. 17, 449-456 (1989).

17. Rennemo, E., Zatterstrom, U. \& Boysen, M. Impact of second primary tumors on survival in head and neck cancer: an analysis of 2,063 cases. Laryngoscope 118, 1350-1356 (2008).

18. Larson, J. T., Adams, G. L. \& Fattah, H. A. Survival statistics for multiple primaries in head and neck cancer. Otolaryngol. Head Neck Surg. 103, 14-24 (1990).

19. Steward, W. P. \& Brown, K. Cancer chemoprevention: a rapidly evolving field. Br. J. Cancer 109, 1-7 (2013).

20. Ang, K. K. et al. Human papillomavirus and survival of patients with oropharyngeal cancer. N. Engl. J. Med. 363, 24-35 (2010).

21. Chaturvedi, A. K. et al. Worldwide trends in incidence rates for oral cavity and oropharyngeal cancers. J. Clin. Oncol. 31, 4550-4559 (2013).

22. Chaturvedi, A. K., Engels, E. A., Anderson, W. F. \& Gillison, M. L. Incidence trends for human papillomavirus-related and -unrelated oral squamous cell carcinomas in the United States. J. Clin. Oncol. 26, 612-619 (2008).

23. Braakhuis, B. J., Visser, O. \& Leemans, C. R. Oral and oropharyngeal cancer in The Netherlands between 1989 and 2006: increasing incidence, but not in young adults. Oral. Oncol. 45, e85-e89 (2009).

24. Marur, S., D'Souza, G., Westra, W. H. \& Forastiere, A. A. HPV-associated head and neck cancer: a virus-related cancer epidemic. Lancet Oncol. 11, 781-789 (2010).

25. Morris, L. G., Sikora, A. G., Patel, S. G., Hayes, R. B. \& Ganly, I. Second primary cancers after an index head and neck cancer: subsite-specific trends in the era of 
human papillomavirus-associated oropharyngeal cancer. J. Clin. Oncol. 29, 739-746 (2011).

26. Cronin, K. A. et al. Annual report to the nation on the status of cancer, part I: national cancer statistics. Cancer 124, 2785-2800 (2018).

27. Warren, S. \& Gates, O. Multiple primary malignant tumors: a survey of the literature and statistical study. Am. J. Cancer 16, 1358-1414 (1932).

\section{ACKNOWLEDGEMENTS}

Preliminary analyses and portions of this data were presented as an oral presentation at the 2017 American Society of Radiation Oncology (ASTRO) Annual Meeting, San Diego and in poster form at the 2018 Multidisciplinary Head and Neck Symposium, Scottsdale.

\section{AUTHOR CONTRIBUTIONS}

S.P.N.: Conceptualization, data curation, formal analysis, investigation, methodology, project administration, visualization, and writing —original draft—review and editing. C.P. III: data curation, investigation, visualization, and writing-review and editing. J. B.: data curation, and writing - review and editing. Z.A.: data curation and writing review and editing. M.K.: data curation and writing-review and editing. A.S.G.: investigation, methodology, and writing - review and editing, H.B.: data curation, investigation, and writing-review and editing, G.B.G.: resources and writingreview and editing, S.J.F.: resources and writing -review and editing, H.D.S.: resources and writing - review and editing, J.P.: resources and writing - review and editing, W. H.M.: resources, methodology and writing - review and editing, R.F.: resources and writing - review and editing, J.M.J.: resources and writing-review and editing, A.S.R. M.: resources and writing-review and editing, S.Y.L.: resources and writing-review and editing, E.M.S.: resources and writing-review and editing, C.D.F.: project administration, supervision, visualization, formal analysis, resources, and writingreview and editing, D.I.R.: supervision, resources, writing-review and editing.

\section{COMPETING INTERESTS}

S.P.N. is funded by the Australian Postgraduate Award, RSNA Fellow Grant, and RANZCR research grants. Dr. C.D.F. is a Sabin Family Foundation Fellow. C.D.F. receives funding and salary support from the National Institutes of Health (NIH), including: the National Institute for Dental and Craniofacial Research Establishing Outcome Measures Award (1R01DE025248/R56DE025248) and an Academic Industrial Partnership Grant (R01DE028290); a National Science Foundation (NSF), Division of Mathematical Sciences, Joint NIH/NSF Initiative on Quantitative Approaches to Biomedical Big Data (QuBBD) Grant (NSF 1557679); a National Institute of Biomedical Imaging and Bioengineering (NIBIB) Research Education Programs for Residents and
Clinical Fellows Grant (R25EB025787-01); the NIH Big Data to Knowledge (BD2K) Program of the National Cancer Institute $(\mathrm{NCl})$ Early Stage Development of Technologies in Biomedical Computing, Informatics, and Big Data Science Award (1R01CA214825); NCl Early Phase Clinical Trials in Imaging and Image-Guided Interventions Program (1R01CA218148); an NIH/NCI Cancer Center Support Grant (CCSG) Pilot Research Program Award from the UT MD Anderson CCSG Radiation Oncology and Cancer Imaging Program (P30CA016672) and an NIH/NCI Head and Neck Specialized Programs of Research Excellence (SPORE) Developmental Research Program Award (P50 CA097007. C.D.F. has received direct industry grant support, honoraria, and travel funding from Elekta AB. Dr. E.M.S. has a research award from Roche Diagnostics supporting HPV testing of oral rinse and mucosal swab samples on the clinical trial for HPV-related cancers screening in men (HOUSTON study).

\section{ADDITIONAL INFORMATION}

Supplementary information is available for this paper at https://doi.org/10.1038/ s41698-019-0097-y.

Correspondence and requests for materials should be addressed to S.P.N. or D.I.R.

Reprints and permission information is available at http://www.nature.com/ reprints

Publisher's note Springer Nature remains neutral with regard to jurisdictional claims in published maps and institutional affiliations.

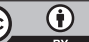

Open Access This article is licensed under a Creative Commons Attribution 4.0 International License, which permits use, sharing, adaptation, distribution and reproduction in any medium or format, as long as you give appropriate credit to the original author(s) and the source, provide a link to the Creative Commons license, and indicate if changes were made. The images or other third party material in this article are included in the article's Creative Commons license, unless indicated otherwise in a credit line to the material. If material is not included in the article's Creative Commons license and your intended use is not permitted by statutory regulation or exceeds the permitted use, you will need to obtain permission directly from the copyright holder. To view a copy of this license, visit http://creativecommons. org/licenses/by/4.0/.

(c) Crown 2019 